\title{
Compass of Research on Ad-hoc Routing Protocols - A Study of the Literature
}

\author{
Rahul Malhotra \\ Adesh Institute of Technology, \\ Gharuan, Punjab, India
}

\author{
Navneet Singh \\ Adesh Institute of Engineering \\ \& Technology, Faridkot, \\ Punjab, India
}

\author{
Sumit Goyal \\ Adesh Institute of Engineering \\ \& Technology, Faridkot, \\ Punjab, India
}

\begin{abstract}
An ad-hoc routing protocol is a caucus, or customary, that controls how nodes decide which way to route packets between computing devices in a mobile ad hoc network. Over the years, abundant routing protocols have been developed for ad hoc mobile networks. These can be categorized into table-driven and on-demand routing. The simulations have shown that there certainly is a need for a special ad-hoc routing protocol when mobility increases and security in mobile ad-hoc networks is an extensive area of research. This research paper is a compassing of research on ad-hoc routing protocols. It gives a sufficient amount of literature for the better understanding of the work ahead.
\end{abstract}

Keywords: Ad-hoc routing; DSR; TORA; AODV; OPNET

\section{INTRODUCTION}

Computer networks have become a lifeline of today's generation. A Wireless Local Area Network is a network using which a mobile user can connect to a Local Area Network (LAN) through a wireless connection. A Wireless Local Area Network can be of type Infrastructure or Ad-hoc. In Infrastructure mode, there must be at least one Wireless Access Point. It acts as a bridge between computers in the wireless network and the computers in the wired networks. An ad-hoc wireless network is one in which various devices send and receive messages to each other in a peer to peer manner. An ad-hoc network tends to feature a small group of devices all in very close proximity to each other. Ad-hoc networks have various characteristics like purpose-specific, autonomous and dynamic. A packet can travel from a source to a destination either directly or through some set of intermediate packet forwarding nodes. Connectivity among nodes varies with time. At a point of time a subset of nodes may be connected and at another point of time, another set of nodes may be connected with each other. Routing is the process of determining the end-to-end path between a source node and a destination node. A routing protocol is needed because it may be necessary to traverse several nodes (multi-hops) before a packet reaches the destination. In wireless networks, due to host mobility, network topology may change from time to time. It is critical for the routing protocol to deliver packets efficiently between source and destination. A routing protocol can be table driven in which each node maintains one or more tables containing routing information to every other node in the network, demand driven in which, routes are created when required or hybrid of both table driven and demand driven. Dynamic Source Routing (DSR) is a demand driven protocol which is designed for use in multi-hop wireless adhoc networks. It makes the network completely selforganizing and self-configuring requiring no existing network infrastructure or administration. Nodes allow communication over multiple hops to exchange data packets among themselves even if they are not in direct wireless transmission range of one another. Temporally-Ordered Routing Algorithm (TORA) is a distributed routing protocol. Routers need to maintain information only about adjacent routers. Like a distance vector routing approach, Temporally-Ordered Routing Algorithm (TORA) maintains route on a perdestination basis. Ad-hoc On-Demand Distance Vector
(AODV) minimizes the number of broadcasts by creating routes on-demand. Each active node periodically broadcasts a Ping message that all its neighbors receive. If a node fails to receive several Ping messages from a neighbor, a link break is detected. In this paper, a review of various demand driven adhoc routing protocols is done.

\section{LITERATURE REVIEW}

Rahul Malhotra et al. [1] did performance analysis of Ad-hoc On-Demand Distance Vector+ (AODV+) over Mac layer based on network parameter throughput. Network Simulation (NS2) was used to create a scenario with 5 mobile nodes. Scenario had both wired and wireless networks. They showed that throughput of receiving packets of Ad-hoc On-Demand Distance Vector+ (AODV+) protocol increases with minimum loss of the packets over the wireless network. The packet receive time of receiver increases with increase in id of receive packets.

S Upadhyay et al. [2] compared three routing protocols Dynamic Source Routing (DSR), Ad-hoc On-Demand Distance Vector (AODV) and Destination-Sequenced Distance-Vector Routing (DSDV) in node mobility and node density increase in the network. As per his findings, Dynamic Source Routing (DSR) routing protocol performed better as compared to Ad-hoc On-Demand Distance Vector (AODV) and Destination-Sequenced Distance-Vector Routing (DSDV) when source node was fixed and destination node was mobile. While keeping the destination node fixed and source node mobile it was again conclude that Dynamic Source Routing (DSR) performance improves much better compared to Adhoc On-Demand Distance Vector (AODV) as well as Destination-Sequenced Distance-Vector Routing (DSDV) routing. A very interesting finding was that when the node density increases then Destination-Sequenced DistanceVector Routing (DSDV) performance deteriorate poorly and it goes nearly to zero value. Also, the performance of Dynamic Source Routing (DSR) routing protocol is much better than Ad-hoc On-Demand Distance Vector (AODV) and Destination-Sequenced Distance-Vector Routing (DSDV). So, under high traffic condition DSR performs well.

S Jain et al.[3] evaluated various ad-hoc routing protocols including Demand Distance Vectoring Routing Protocol (AODV), Dynamic Source Routing (DSR) and Dynamic MANET On demand Routing (DYMO) on the basis of the 
packet delivery ratio, average end to end delay, average jitter and throughput. Conclusion was that complexities of routing between the nodes increase due to the highly dynamic nature of the ad-hoc network. The routing protocols are faced with the challenge of producing multi-hop routing as the host moves.

S Joshi et al. [4] did performance analysis of proactive and reactive routing protocols. Analysis was done for DestinationSequenced Distance-Vector Routing (DSDV) and Ad-hoc OnDemand Distance Vector (AODV). Various constraints related to ad-hoc networks like shared nature of the wireless medium, limited transmission power \& range, node mobility, battery life, bandwidth limitation etc. were considered. Comparison of simulation results concluded that DestinationSequenced Distance-Vector Routing (DSDV) protocol performs better in the small networks with less mobility of nodes. It was also concluded that Ad-hoc On-Demand Distance Vector (AODV) is more adaptable to large scale networks.

Rahul Malhotra and Gurpreet Singh [5] compared the performance of Dynamic Source Routing (DSR) protocol and Temporally-Ordered Routing Algorithm (TORA) protocol for wireless ad-hoc network with 50 nodes using OPNET modeler. Various parameters like traffic sent and received, end to end delay during data communication and download and upload response time have were analyzed. They showed that the Dynamic Source Routing protocol is able to forward $95 \%$ of the traffic received. On the other hand, Temporally Ordered Routing Algorithm protocol forwards only 39\% of the traffic received. Based on results, it was further shown that Dynamic Source Routing protocol has given comparatively better performance than Temporally Ordered Routing Algorithm protocol.

Rahul Malhotra et al. [6] reviewed various features of wireless networks, ad-hoc wireless networks and the routing protocols for wireless ad-hoc networks. They took two on-demand routing protocols Dynamic Source Routing (DSR) and Temporally-Ordered Routing Algorithm (TORA) for the further study. They suggested that routing protocols can be compared for their data throughput, media access delay, upload response time, download response time, traffic sent and traffic received.

Rahul Malhotra and Sheenu Girdher[7] simulated multi hop cellular network and self-organizing packet radio ad-hoc network with overlay and analyzed their performance in terms of inbound, outbound and through traffic, traffic received, sent and dropped at routers. Further they simulated Ethernet delay at every node of Local Area Network (LAN). Multi hop Cellular Network (MCN) network uses cellular and wireless technology whereas Self Organizing Packet Radio Networks with Overlay (SOPRANO) uses cellular and MANET. Based on simulation results they concluded that Self Organizing Packet Radio Networks with Overlay (SOPRANO) reduces interference between nodes when traffic load is very high. Interference problem occurs because all nodes take part to transmit data. In Multi hop Cellular Network (MCN) number of base stations are reduced because data can be transferred with the help of nodes itself.

Rahul Malhotra et al. [8] performed a comparative study of next generation high speed wireless networks. Different Hybrid Wireless Networks which are a combination of ad-hoc wireless networks and cellular networks were studied. They suggested that different parameters considered for the design of a hybrid wireless network can be categorized on the basis of spectrum reusability, overlay, throughput enhancement and data forwarding system used which helps in reduced interference, extended coverage, increased reliability etc.

Rahul Malhotra et al. [9] compared various ad-hoc routing protocols based on Good put and Routing Load. Two protocols, On-Demand Distance Vector (AODV) and Destination-Sequenced Distance Vector (DSDV) were taken up for study based on stated parameters. They showed that good put is higher in the case of Destination-Sequenced Distance Vector (DSDV) as compare to On-Demand Distance Vector (AODV) due to re-transmission of the packets, which has been lost due to noise, error and some congestion. The total routing load of packet in AODV protocol is higher than of Destination-Sequenced Distance Vector (DSDV) protocol. This means the chances of congestion are more in the case of On-Demand Distance Vector (AODV) protocol.

Rahul Malhotra and Karandeep Singh[10] implemented core based tree protocols for Mobile ad-hoc networks and analyzed different network parameters viz. bandwidth, delay, traffic sent, traffic received and traffic dropped. They proved that when network load is increased, the network bandwidth is utilized in an appropriate manner due to the increase in number of packets. Further, when the nodes are increased according to the capacity of the network medium, the rate of transfer in the network starts decreasing, that is the delay; network load and traffic in the networks tend to increase.

Rahul Malhotra et al. [11] studied table driven protocols for ad-hoc wireless networks. They analyzed Distributed Bellman-Ford Algorithm (DBF) and Dynamic Source Routing (DSR) protocol. Based on simulation results, they showed the on demand routing protocol, Dynamic Source Routing (DSR) gives better performance when compared with Table-driven routing protocol, Distributed Bellman Ford (DBF). From the above conclusions, it is concluded that the On Demand routing protocols based Dynamic Source Routing (DSR) technique gives larger bandwidth, lesser delay and provides better control overhead than Distributed Bellman Ford (DBF) technique based table driven routing protocols.

Ullah et al. [12] simulated on the reliability of ad-hoc routing protocols for loss-and-delay sensitive applications. They showed that the reliability function of such a multipath system is concave with respect to the total number of paths. They proved that a partially-disjoint path is more reliable than a node-disjoint path. They analyzed the impact of multiple node disjoint paths on the reliability of a typical ad-hoc routing system in the context of delay-and-loss sensitive applications and also proved that the reliability of multiple paths increases exponentially with the addition of first few paths and then saturates at a steady-state value.

Rahul Malhotra et al. [13] analyzed core migration protocols in wireless ad-hoc networks by adopting multiple nodes diverse test-bed and studied core selection. Three case studies were done, first for 5 nodes, second for 7 nodes and third for 10 nodes. They verified the results for the core migration in wireless ad hoc networks obtained with $\mathrm{C}++$ platform with that of the JAVA platform and it was concluded that the core migration achieved with the JAVA programming results into the optimal location of the core than that obtained from the network graph modeled using $\mathrm{C}++$.

Rahul Malhotra et al. [14] threw light on operational aspects of various techniques of core selection and core migration in wireless ad-hoc networks. They discussed multicast routing based on centroid based core selection and random core 
selection. They suggested that core migration in ad-hoc networks is invoked due to recovery from core failure and core degeneration and migration.

R Ramdhany et al.[15] described in paper, "Dynamic deployment and reconfiguration of ad-hoc routing protocols," MANET Kit by showing how it can be used to straightforwardly build and dynamically deploy major ad-hoc routing protocols and how these deployments can be variegated in a number of ways to suit different operating conditions. Furthermore, empirical evaluation showed that MANET Kit meets stated goals by achieving comparable performance to monolithic implementations of the same protocols, achieving smaller resource overheads when more than one protocol is implemented in comparison to the monolithic approach, and also achieving significant code reuse across protocols.

M Frikha et al. [16] described in Load-balancing in MANET shortest-path routing protocols that load-balancing mechanisms that push the traffic further from the center of the network. Basically, they provide novel routing metrics that take into account nodes degree of centrality, for both proactive and reactive routing protocols. They proposed loadbalancing schemes that push the traffic further from the center of the network. To define central node, we used two characterizations, depending on whether the routing protocol is proactive or reactive. For nodes using reactive approaches, they characterized their centrality by the size of their routing tables. As for proactive approaches, a node's centrality was defined by size of its MPR Selector List. Subsequently, routing metrics minimizing the average route centrality were accordingly proposed and implemented on two representatives of reactive and proactive approaches, respectively Ad-hoc OnDemand Distance Vector (AODV) and OLSR.

J Matthew [17], proposed in" Ad-hoc routing for multilevel power save protocols" a link layer technique and routing protocol that adapts to an application-defined latency in an energy-efficient manner. They described placing nodes in different power save states that tradeoff energy consumption and latency. Their adaptive sleeping technique allows nodes to adjust their sleeping interval in response to the desired latency of data that it is forwarding. They evaluated their protocols via simulation and find that they allow end-to-end latency bounds to be achieved with much less energy consumption than turning power saves off. Their technique can maintain a desired latency bound with only a small increase in energy consumption over traditional power save protocols and with far less energy consumption than turning power save off.

Jiangchua Wen et al.[18] simulated An adaptive fuzzy logic based secure routing protocol in mobile ad-hoc networks They proposed algorithm of Security-Level is an adaptive fuzzy logic based algorithm that can adapt itself with the dynamic conditions of mobile hosts. They presented a Fuzzy Logic Based Secure multicast routing protocol (FLSL) routing protocol for MANETs. An interesting property is that every node in the MANET has the field of Security-Level based on the fuzzy logic in the route tables to select the highest Security-Level route. The Fuzzy Logic Based Secure multicast routing protocol (FLSL) routing protocol can improve MANET's security. It is feasible to the weak security character of mobile ad-hoc networks.

P Peter, Perreau [19] proposed a new routing protocol which increases the network throughput. The protocol is a multi-path routing protocol with a load balance policy. The simulations show a significant improvement in terms of connection throughput and end-to-end delay, when compared to singlepath routing. They also explained theoretical analysis allowing to compare reactive single-path and multi-path routing with load balance mechanisms in ad-hoc networks, in terms of overheads, traffic distribution and connection throughput.

F Bai et al. [20] discussed the important framework for analyzing the impact of mobility on performance of routing protocols for ad-hoc networks, there framework aims to evaluate the impact of different mobility models on the performance of MANET routing protocols. They proposed various protocol independent metrics to capture interesting mobility characteristics, including spatial and temporal dependence and geographic restrictions. They showed that the protocol performance may vary drastically across mobility models and performance rankings of protocols may vary with the mobility models used.

\section{STATEMENT OF PROBLEM}

The objective of this study is to understand various parameters of Dynamic Source Routing (DSR) and Temporally-Ordered Routing Algorithm (TORA).Various parameters of focus are inbound and outbound traffic through Local Area Network (LAN) using Dynamic Source Routing (DSR), traffic received \& forwarded through switch using Dynamic Source Routing (DSR), traffic sent, received and dropped through routers using Dynamic Source Routing (DSR), comparison of delay results at every node of Local Area Network (LAN) using Dynamic Source Routing (DSR), Ethernet load, traffic received and delay through various nodes using Dynamic Source Routing (DSR), traffic received and forwarded in central switch using Temporally-Ordered Routing Algorithm (TORA), traffic received and forwarded in network using Temporally-Ordered Routing Algorithm (TORA), traffic sent, received and dropped in routers using Temporally-Ordered Routing Algorithm (TORA).

\section{NETWORK SIMULATION}

Network simulation is a method of modeling the behavior of a network to study it. Interaction between different components of a network is calculated using mathematical formulas or by capturing and playing back observations from an actual network. The behavior of the network and the various applications and services it supports can then be observed in a test lab. Different attributes of the environment can also be modified in a controlled manner to assess how the network would behave under different conditions.

OPNET is a network simulation tool for modeling, simulating and analyzing the performance of communication networks, distributed systems, computer systems and applications. It comes with different toolsets. Node model specifies interface of a network component, packet format defines protocols, process model abstracts the behavior of a network component, and project window defines network topology and link connections and simulation window captures and displays simulation results.

Network Simulator, widely known as NS2, is an event driven simulation tool that has proved useful in studying the dynamic nature of communication networks. Simulation of wired as well as wireless network functions and protocols can be done using NS2. It contains modules for numerous network components such as routing, transport layer protocol, application, etc. To investigate network performance, 
researchers can simply use an easy-to-use scripting language to configure a network and observe results generated by NS2.

OMNeT is another network simulation environment. It is an open source environment which provides a component based architecture for models. Components are programmed in $\mathrm{C}++$, and then assembled into larger components and models using a high-level language. Reusability of models is there. OMNeT has extensive GUI support. It is gaining importance in network simulation due to its modular nature.

In this work, we have used OPNET modeler because it offers relatively much powerful visual or graphical support for the users. It is commercial software. The graphical editor interface can be used to build network topology and entities from the application layer to the physical layer. Objectoriented programming technique is used to create the mapping from the graphical design to the implementation of the real systems. It is based on a mechanism called discrete event system which means that the system behavior can simulate by modeling the events in the system in the order of the scenarios the user has set up. Hierarchical structure is used to organize the networks. OPNET also provides programming tools for users to define the packet format of the protocol. The programming tools are also required to accomplish the tasks of defining the state transition machine, defining network model and the process module. Three main functions of OPNET are modeling i.e. to model the network consideration, simulating i.e. perform the actual simulation to get the results and analysis to analyze various parameters and results.

\section{CONCLUSION}

This paper gives an overview of various features of wireless networks, ad-hoc wireless networks and the routing protocols for wireless ad-hoc networks. Based on literature review we are trying to simulate and analyze Dynamic Source Routing (DSR) and Temporally-Ordered Routing Algorithm (TORA) for various parameters like Inbound and outbound traffic through Local Area Network (LAN), traffic received \& forwarded through switch, traffic sent, received and dropped through routers, comparison of delay results at every node of Local Area Network (LAN) and Ethernet load, traffic received and delay through various nodes.

\section{REFERENCES}

[1] Rahul Malhotra, Sangeeta Monga, Kamaljeet Kaur, (2012), "Performance Analysis of AODV+ Over Mac Layer Based on Throughput", Journal of Computing Vol. 4(1).

[2] S Upadhyay, N Gandotra, P Joshi and A Kumar (2012), "Comparison and performance analysis of reactive type DSR, AODV and proactive type DSDV routing protocol for wireless mobile ad-hoc network", Journal of Engineering and Computer Innovations Vol. 2(10), pp. 36-47.

[3] S Jain, V Kumar and S Tiwari, (2012), "Impact of Node Density and Mobility on Scalable Routing Protocols in Mobile Ad-Hoc Networks", IJCA Special Issue on Communication Security comnetcs vol.1, pp. 21-27. Fröhlich, B. and Plate, J. 2000. The cubic mouse: a new device for three-dimensional input. In Proceedings of the SIGCHI Conference on Human Factors in Computing Systems.

[4] S Joshi, S Kurundkar and L Waghmare, (2012), "Performance Analysis of Routing Protocol With Mobility Constraint", IJCA Proceedings on International Conference and workshop on Emerging Trends in

www.ijsea.com
Technology icwet vol.7, pp. 13-16. Sannella, M. J. 1994 Constraint Satisfaction and Debugging for Interactive User Interfaces. Doctoral Thesis. UMI Order Number: UMI Order No. GAX95-09398., University of Washington.

[5] Rahul Malhotra, Gurpreet Singh, (2011), "Simulation Analysis of DSR and TORA: On Demand Wireless Ad-hoc Routing Prot o c o ls", Journal of Computing Vol. 3(8).

[6] Rahul Malhotra, Gurpreet Singh, (2011), “An Overview of on demand Wireless ad-hoc network protocols: DSR and TORA", International Journal of Computer Technology and Applications Vol. 3(8), pp. 1641-1651.

[7] Rahul Malhotra, Sheenu Girdher, (2011), "Investigation of Multi-hop Cellular Network and Self organizing Packet Radio Next Generation Hybrid Wireless Networks," International journal of advanced engineering sciences and technologies (IJAEST), Vol. 8(1), pp.025-031.

[8] Rahul Malhotra, Sheenu Girdher, (2011), "Comparative study of next generation high speed wireless network", Indian Journal of Computer Science and Engineering, Vol. 2(3), pp. 391-401.

[9] Rahul Malhotra, Sangeeta Monga, Gurmeet Kaur, (2011), "Comparison of AdHoc Routing Protocols on the Basis of Good put and Routing Load", Journal of Computing, Vol. 3(12).

[10] Rahul Malhotra, Karandeep Singh, (2011), "Implementation of Core Based Tree Protocols for Mobile Adhoc Networks", Journal of Computing, Vol. 3(6).

[11] Rahul Malhotra, Nitin Nikesh, Sangeeta Monga, (2011), "Analysis of Table Driven and On-demand Routing Protocols for Mobile Adhoc Networks ", Indian Journal of Computer Science and Engineering, Vol. 2(3), pp. 391-401.

[12] Ullah, S Ali, M Farooq and S Muhammad, (2011), "On the reliability of ad hoc routing protocols for loss-anddelay sensitive applications", Ad Hoc Networks 9, pp.285-299.

[13] Rahul Malhotra, Reena Aggarwal, Sangeeta Monga, (2010), "Analyzing Core Migration Protocol Wireless Ad Hoc Networks by Adopting Multiple Nodes Diverse Test-bed", International Journal on Emerging Technologies, Vol. 2(1), pp. 1-6.

[14] Rahul Malhotra, Reena Aggarwal, Sangeeta Monga, (2010), "Analyzing Core Migration Protocol Wireless Ad Hoc Networks by Adopting Multiple Nodes Diverse Test-bed", International Journal of Engineering Science and Technology, Vol. 2(10), pp. 5326-5331.

[15] R Ramdhany, G Coulson, P Grace and D Hutchison, (2010), "Dynamic deployment and reconfiguration of adhoc routing protocols", Internet Serv Appl 1, pp. 135152.

[16] M Frikha, B Hamouda and O Souihli, (2009), "Loadbalancing in MANET shortest-path routing protocols", Ad Hoc Networks 7, vol pp. 431-442.

[17] J Matthew, Nitin and H Vaidya, (2008), "Ad hoc routing for multilevel power save protocols", Ad Hoc Networks vol 6, pp. 210-225. 
[18] JiangchuaWen, N Ji, J Nie, Xin He and Zheng Zhou, (2006) , "An adaptive fuzzy logic based secure routing protocol in mobile ad hoc networks", Fuzzy Sets and Systems vol 157, pp. $1704-1712$.

[19] V Aline, M Dias, De Amorim, S Fdida and J Ferreira, (2005), "Self-organization in spontaneous networks: the approach of DHT-based routing protocols", Ad Hoc Networks vol 3, pp. 589-606.

[20] P Peter and P Sylvie, (2004), "Increasing the network performance using multi-path routing mechanism with load balance", Ad Hoc Networks vol 2, pp. 433-459.

[21] F Bai and S Narayanan, (2003), "The important framework for analyzing the Impact of Mobility on Performance Of Routing protocols for Ad hoc Networks", Ad Hoc Networks vol 1, pp. 383-403.

[22] M. R. Elizabeth and E Perkins,(2003), "Evolution and future directions of the ad hoc on-demand distancevector routing protocol", Ad Hoc Networks vol 1, pp.125-150.

[23] Z Gil Z and A Segall, (2003), "Energy efficient routing in ad hoc disaster recovery networks", Ad Hoc Networks vol 1, pp. 405-421.

[24] P Papadimitratos and J Zygmunt, (2003), "Secure message transmission in mobile ad hoc networks", Ad Hoc Networks vol 1, pp. 193-209.

[25] S Lee, C Kim, (2002), "A new wireless ad hoc multicast routing protocol", Journal of Computer Networks and Isdn Systems on Computer Networks vol. 38, no. 2, pp. 121-135.

[26] J Liu, S Singh, (2001), “ATCP: TCP for Mobile Ad Hoc Networks", IEEE Journal on selected in communication, vol. 19, no. 7, pp. 1506-1531.

[27] H Zhou, S Singh , (1998), "Content based multicast $(\mathrm{CBM})$ in ad hoc networks", Journal on Routing in Mobile Communication Networks, vol. 1, no. 2, pp. 246257.

[28] P Kuosmanen ,(1997), "Classification of Ad Hoc Routing Protocols", Journal of Mobile Networking and Computing Systems, vol. 4, no. 2, pp. 103-139.

[29] Mobile ad hoc networks (MANET). Http: //www.ietf.org/html.charters/manetcharter.html, 1997, IETF Working Group Charter.

[30] Scott C and Vincent D, "A highly adaptive distributed routing algorithm for mobile wireless networks", Proceedings of INFOCOM 1997, 1997. 